\title{
Submersion injury in children: a Hong Kong perspective
}

\author{
KL Hon *, MD, FAAP, Karen KY Leung, MB, BS, MRCPCH, Jeff CP Wong, MB, BS, MRCPCH \\ Department of Paediatrics and Adolescent Medicine, The Hong Kong Children's Hospital, Hong Kong \\ *Corresponding author: ehon@cuhk.edu.hk
}

Hong Kong Med J 2020;26:540-2

https://doi.org/10.12809/hkmj198129

Submersion injury (SI) is one of the three most common causes of childhood accidental fatalities worldwide, accounting for approximately $28 \%$ of them. ${ }^{1}$ According to the World Health Organization's World Report on Child Injury Prevention, the rate of SI fatalities among children is 7.2 deaths per 100000 population worldwide, and 1.2 per 100000 population in high-income countries. ${ }^{1}$ As per the Childhood Injuries and Injury Surveillance in Hong Kong, there were 43 fatalities due to accidental SI among those aged $<19$ years from 2001 to 2009 , which is $7 \%$ of all deaths of external cause for that age-group. ${ }^{2}$ In Hong Kong, SI is uncommon, with a low death rate of 0.34 per 100000 population. $^{2}$ According to the Leisure and Culture Service Department's 2016 to 2018 special incidents statistics, there has been a decline in the number of incidents of rescue and SI in public swimming pools and beaches in Hong Kong. ${ }^{3}$ In 2018 , there were only four deaths due to SI in public swimming pools and beaches. ${ }^{3}$ The authors, having managed two cases of SI in July 2019, would like to raise awareness of paediatric SI in Hong Kong and potential public health implications.

\section{Hong Kong experience}

Most deaths due to SI in Hong Kong occur in natural bodies of water; followed by those that occur in swimming pools. ${ }^{2,4-6}$ Some patients with SI occurring in mainland China are transported to Hong Kong for treatment. In our paediatric experience, these patients appear more likely to incur SI indoors and are generally younger, have lower Glasgow Coma Scale score, present with asystole, and require intubation at the emergency department. ${ }^{7}$ The risk of fatal SI is higher among tourists, likely related to the increased exposure to water when on holiday and to the unfamiliar environment. ${ }^{1,8,9}$ Incidents of SI among tourists are not reported in Hong Kong.

The authors recently experienced two SI cases involving tourists visiting Hong Kong. The first case involved a boy with good past health. The patient was near-drowned in a public swimming pool where he received on-scene cardiopulmonary resuscitation (CPR) by the lifeguard on duty. He had a return of spontaneous circulation after a long duration of resuscitation but subsequently developed multiorgan dysfunction and died. The second patient was a teenage girl with good past health. She was neardrowned in the sea and developed acute respiratory distress syndrome. Both the caregiver and the girl were inexperienced swimmers. She required intensive care and ventilatory support and survived.

\section{Prevention of submersion injuries}

Understanding the risk factors can promote effective prevention of SI. Risk factors for SI included male sex, age $<5$ years, ethnic minority status, and presence of underlying health conditions including epilepsy, certain cardiac arrhythmia, or autism. ${ }^{1}$ Environmental factors include exposure to open water, lack of supervision, lack of available personal floatation devices or lifeguards, lack of barriers to prevent children dropping into water, and uneven, slippery or steep surfaces near or in water. ${ }^{1}$ The Child Fatality Review Final Report in 2010 concluded that two out of three deaths due to SI occurred because the child swam in places not suitable for swimming and had overestimated their swimming ability. ${ }^{10}$ Public education should emphasise vigilant adult supervision during swimming at all times and wearing personal floatation devices around water. ${ }^{11}$ Children should also be encouraged to attend swimming classes at an early age for basic swimming skills. Participation in formal swimming lessons is associated with an $88 \%$ reduction in the risk of SI among children aged 1 to 4 years. ${ }^{12}$

To preventing SI in natural water, public education should focus on discouraging swimming in areas that are unsuitable or unsafe for swimming, such as lakes and ponds, and understanding and following public notices, such as beach warning flags. The public should also be reminded to check the weather forecast before swimming or participating in any water activities, and to leave the water immediately when there is thunder or lightning. ${ }^{13}$

To prevent SI in swimming pools, pool design can be reviewed. The Leisure and Culture Services Department should consider regular audits of private and public swimming pools to ensure that they meet safety standards. Fencing and security 
gates can prevent unauthorised access to swimming pools. Technological methods may be considered to supplement present preventive measures against SI. For example, tele-supervision with multiple video cameras around swimming pools or beaches, alarms that can detect individuals who are motionless under the water for 1 to 2 minutes, or wireless heart rate monitors for children in public swimming pools.

To prevent indoor SI, buckets or other vessels should be emptied of water if toddlers are around, and top-loading washing machines should not be filled with water when it is not in operation. ${ }^{4}$ Young children should not be taken to spas and hot tubs; when not in use, pools and tubs should be covered.

\section{Improving outcomes of submersion injuries}

The presence of lifeguards may not be sufficient to prevent SI and related deaths. Prompt effective CPR by bystanders may improve the chances of survival. The Hong Kong Life Saving Society is the only body recognised by the International Life Saving Federation for assessing and awarding lifeguard qualifications in Hong Kong. Although the training of the lifeguards in Hong Kong includes Basic Life Support and automated external defibrillation, these qualifications are only reviewed every 3 years. The health authorities should consider arranging free refresher courses and simulation training, auditing lifeguards' ability to perform CPR, and ensuring the availability of necessary medical equipment, especially in private swimming pools.

\section{Potential public health implications}

\section{Financial implications}

Both cases involved tourists in Hong Kong and were non-entitled person under the Hospital Authority System. Intensive care support is expensive, and the cost is nearly HK\$30000 per day. Social department has protocols to waive some of these payments, but it is not guaranteed to all cases. The judgement call will be difficult and strict transferral criteria will need to be in place to prevent the abuse of this system.

\section{Brain death and organ donation}

One of the cases had sustained a very significant insult to the brain, therefore the issues of brain death and organ donation were discussed. The public has increased acceptances about the concept of brain death legally equating cardiopulmonary death over the past two decades, however it is still not ideal. The diagnosis of paediatric brain death is seldom made, making organ donation exceedingly difficult in Hong Kong. ${ }^{14}$

\section{Burial issues and aviation transportation of corpse}

The non-survived case is from a different cultural background. Some religions prohibit postmortem investigations and demand burial rituals to be completed within a short period of time following death. Family members may request repatriation of the remains. Liaisons with legal department and airliner are needed to facilitate the process. According to The International Air Transport Association Guidelines, the corpse has to be packed in a hermetically sealed inner containment, inside a wooden or metal coffin. ${ }^{15}$ The cost can be significant; cremated remains might be an alternative if it is accepted by the family.

\section{Conclusion}

Most SIs are preventable. Primary prevention by health promotion and public health measures to prevent SI can save lives. Prompt and effective onscene CPR can improve survival and outcomes for patients with SI.

\section{Author contributions}

All authors contributed to the concept or design, acquisition of the data, analysis or interpretation of the data, drafting of the manuscript, and critical revision of the manuscript for important intellectual content. All authors had full access to the data, contributed to the commentary, approved the final version for publication, and take responsibility for its accuracy and integrity.

\section{Conflicts of interest}

As an editor of the journal, KL Hon was not involved in the peer review process. The other authors have no conflicts of interest to disclose.

\section{Funding/support}

This commentary received no specific grant from any funding agency in the public, commercial, or not-for-profit sectors.

\section{References}

1. Peden M, Oyegbite K, Ozanne-Smith J, et al. World Report on Child Injury Prevention. Geneva: World Health Organization; 2008.

2. Chow CB. Childhood injuries and injury surveillance in Hong Kong. Available from: https://www.fhs.gov.hk/ english/archive/MCHconference2012/presentations/21. pdf. Accessed 2 Sep 2019.

3. Beaches and Swimming Pools, Leisure and Culture Services Department, Hong Kong SAR Government. Statistics on special incidents. 2018. Available from: https://www.lcsd. gov.hk/en/beach/atten-general/atten-stat.html. Accessed 4 Sep 2019.

4. Hon KL, Leung TF, Chan SY, Cheung KL, Ng PC. Indoor versus outdoor childhood submersion injury in a densely populated city. Acta Paediatr 2008;97:1261-4.

5. Hon KL, Leung AK. Childhood accidents: injuries and 
poisoning. Adv Pediatr 2010;57:33-62.

6. Law AK, Ng MH, Hon KL, Graham CA. Out-of-hospital cardiac arrest in the pediatric population in Hong Kong: a 10-year review at a university hospital. Pediatr Emerg Care 2018;34:179-84.

7. Hon KL, So KW, Wong W, Cheung HM, Cheung KL. Radiologic, neurologic and cardiopulmonary aspects of submersion injury. Pediatr Emerg Care 2016;32:623-6.

8. Mackie IJ. Patterns of drowning in Australia, 1992-1997. Med J Aust 1999;171:587-90.

9. Morgan D, Ozanne-Smith J, Triggs T. Descriptive epidemiology of drowning deaths in a surf beach swimmer and surfer population. Inj Prev 2008;14:62-5.

10. Social Welfare Department, Hong Kong SAR Government. Review Panel of the Pilot Project on Child Fatality Review Final Report. 2010. Available from: https://www.swd.gov. hk/en/index/site_pubsvc/page_family/sub_fcwdocument/ id_cfrp/. Accessed 1 Aug 2019.

11. Food and Health Bureau, Hong Kong SAR Government. Hong Kong Reference Framework for Preventive Care for Children in Primary Care Settings. 2012. Available from: https://www.fhb.gov.hk/pho/english/health professionals/professionals_preventive_children_pdf. html. Accessed 1 Aug 2019.

12. Brenner RA, Taneja GS, Haynie DL, et al. Association between swimming lessons and drowning in childhood: a case-control study. Arch Pediatr Adolesc Med 2009;163:203-10.

13. Leung TH. Non-communicable Disease Watch. Epidemiology and prevention of unintentional drowning. 2012. Available from: https://www.chp.gov.hk/files/pdf/ ncd_watch_jul2012.pdf. Accessed 2 Sep 2019.

14. Hon KL, Poon TC, Wong W, et al. Prolonged non-survival in PICU: does a do-not-attempt-resuscitation order matter. BMC Anesthesiol 2013;13:43.

15. International Air Transport Association. Handling of Human Remains. IATA Airport Handling Manual. 2011:155. Available from: https://flexmort.com/wpcontent/uploads/2013/06/IATA-Richtlinie-.-s.-155AHM-333.pdf. Accessed 1 Aug 2019. 